\title{
Identification of the Corticobulbar Tracts of the Tongue and Face Using Deterministic and Probabilistic DTI Fiber Tracking in Patients with Brain Tumor
}

\author{
M. Jenabi, (D)K.K. Peck, (D)R.J. Young, N. Brennan, and (D) A.I. Holodny
}

\begin{abstract}
BACKGROUND AND PURPOSE: The corticobulbar tract of the face and tongue, a critical white matter tract connecting the primary motor cortex and the pons, is rarely detected by deterministic DTI fiber tractography. Detection becomes even more difficult in the presence of a tumor. The purpose of this study was to compare identification of the corticobulbar tract by using deterministic and probabilistic tractography in patients with brain tumor.
\end{abstract}

MATERIALS AND METHODS: Fifty patients with brain tumor who underwent DTI were studied. Deterministic tractography was performed by using the fiber assignment by continuous tractography algorithm. Probabilistic tractography was performed by using a Monte Carlo simulation method. ROls were drawn of the face and tongue motor homunculi and the pons in both hemispheres.

RESULTS: In all subjects, fiber assignment by continuous tractography was ineffectual in visualizing the entire course of the corticobulbar tract between the face and tongue motor cortices and the pons on either side. However, probabilistic tractography successfully visualized the corticobulbar tract from the face and tongue motor cortices in all patients on both sides. No significant difference $(P<.08)$ was found between both sides in terms of the number of voxels or degree of connectivity. The fractional anisotropy of both the face and tongue was significantly lower on the tumor side $(P<.03)$. When stratified by tumor type, primary-versus-metastatic tumors, no differences were observed between tracts in terms of the fractional anisotropy and connectivity values $(P>.5)$.

CONCLUSIONS: Probabilistic tractography successfully reconstructs the face- and tongue-associated corticobulbar tracts from the lateral primary motor cortex to the pons in both hemispheres.

ABBREVIATIONS: $\mathrm{CBT}=$ corticobulbar tract; $\mathrm{FA}=$ fractional anisotropy; $\mathrm{FACT}=$ fiber assignment by continuous tractography

$\mathbf{P}$ recise localization of white matter fibers adjacent to brain tumors, such as the corticospinal tracts, is important for presurgical planning to maximize resection of brain lesions and minimize iatrogenic complications. ${ }^{1,2}$ Diffusion tensor imaging is a rapidly growing technique to visualize the connectivity of white matter networks insufficiently evaluated by other imaging modalities. $^{2-4}$ The DTI technique is based on the anisotropic diffusion of water that preferentially occurs along the longitudinal axis of axons, which allows the delineation of white matter from the diffusion patterns of water molecules. ${ }^{4,5}$

Received December 5, 2014; accepted after revision March 26, 2015.

From the Departments of Radiology (M.J., K.K.P., R.J.Y., N.B., A.I.H.) and Medical Physics (K.K.P.) and Brain Tumor Center (R.J.Y., A.I.H.), Memorial Sloan-Kettering Cancer Center, New York, New York; and Department of Radiology (R.J.Y., A.I.H.), Weill Medical College of Cornell University, New York, New York.

Please address correspondence to Andrei I. Holodny, MD, Department of Radiology, Memorial Sloan-Kettering Cancer Center, 1275 York Ave, NY, NY 10021; e-mail: holodnya@mskcc.org

三 Indicates article with supplemental on-line table.

http://dx.doi.org/10.3174/ajnr.A4430
The DTI data can be reconstructed via tractography algorithms to display the anatomic localization of the entire white matter. ${ }^{4,6-13}$ The algorithms have been classified into 2 types: deterministic (streamline) and probabilistic. The fiber assignment by continuous tractography (FACT) algorithm is a popular deterministic method in which the propagation of tracking is primarily determined by the principal eigenvector of fractional anisotropy (FA) of each voxel. The measurement of FA is highly dependent on the distributions and intactness of axonal membranes and myelin. However, FACT is unable to solve fiber-crossing problems. The FA decreases in axonal regions with complex architecture due to crossing fibers, intricate branching configurations, and modified tissue organization due to nearby tumors. ${ }^{1,3,12,14}$ One method to overcome these problems is the probabilistic tracking algorithms. ${ }^{6,9}$ The probabilistic approach considers multiple fiber connectivity from large numbers of potential pathways to generate a connectivity map with the most probable connected voxels between seed points.

Visualizing the corticobulbar tract (CBT) associated with the 
face and tongue by using FACT is a special challenge because of the relatively small size of the CBT and the presence of prominent crossing fibers-the longitudinal fasciculus. ${ }^{4,6,8}$ The CBT forms part of the descending pyramidal tract, in conjunction with the corticospinal tract. It originates from the primary motor cortex and descends through the corona radiata and internal capsule into the cerebral peduncles. The CBT then innervates the motor cranial nerve nuclei in the brain stem to control the muscles of the face, head, and neck. Studies based on single tensor deterministic tractography have failed to identify the CBT fibers associated with the face and tongue. ${ }^{12,15-18}$ There have been a few, relatively sophisticated, high-angular-resolution diffusion imaging-based multitensor techniques with some success in extracting fibers from wider areas of the primary motor cortex, including the face and tongue areas ${ }^{15,17,18}$; however, high-angular-resolution diffusion imaging-based techniques have not been applied to large cohorts of patients with brain tumors. Multitensor techniques also require long acquisition times, which are a limiting factor in patients with brain tumors and neurologic deficits.

The purpose of this study was to compare the detection of the CBT fibers associated with the face and tongue by using both deterministic and probabilistic tractography in patients with brain tumors. Given that the probabilistic approach allows the visualization of a distribution of multiple fiber connectivity, we hypothesized that probabilistic tractography will outperform deterministic tractography.

\section{MATERIALS AND METHODS \\ Patients}

This retrospective study was approved by the local institutional review board. Fifty consecutive patients $7-80$ years of age (20 males; mean age, 56.2 years; 30 females, mean age, 57.1 years), with single unilateral tumors, who underwent DTI as part of their standard of care during a 12-month period were studied. DTI was performed whenever the margin of the enhancing tumor or peritumoral FLAIR/T2 abnormality or both were within $2 \mathrm{~cm}$ of the face and/or tongue motor cortex and/or descending CBT on anatomic images. In none of the cases did the tumor (defined as abnormal enhancement or abnormal findings on FLAIR/T2) involve the contralateral side. The tumors consisted of glioblastomas $(n=17)$, metastases $(n=19)$, astrocytomas $(n=6)$, anaplastic astrocytomas $(n=3)$, oligoastrocytomas $(n=3)$, oligodendroglioma $(n=1)$, and hemangiopericytoma $(n=1)$. Among the 50 patients, only 6 received treatments before DTI. Five had chemotherapy and radiation therapy, and 1 had chemotherapy only.

\section{MR Imaging Acquisition and Analysis}

DTI was performed on 3T magnets (Signa Excite, Signa HDx, and Discovery 750HD; GE Healthcare, Milwaukee, Wisconsin) by using an 8-channel head coil. Data were acquired by using a singleshot spin-echo echo-planar imaging sequence $(\mathrm{TR} / \mathrm{TE}=11,000$ and $13,500 / 60$ and $100 \mathrm{~ms}$, matrix $=128 \times 128 \mathrm{~mm}$, section thickness $=3 \mathrm{~mm}$, b-value $=1000 \mathrm{~s} / \mathrm{mm}^{2}$, FOV $=240 \mathrm{~mm}$, in-plane resolution $=1.8 \times 1.8 \times 3 \mathrm{~mm}$ with 25 noncollinear gradient directions). Head motion and eddy current corrections were applied to minimize artifacts; DTI\&FiberTools (University Hospital, Freiburg, Germany) DTI analysis software imple- mented in Matlab (MathWorks, Natick, Massachusetts), was used to analyze DTI data. Routine anatomic images including axial 3D T1-weighted images by using a spoiled gradient-recalled-echo sequence $(\mathrm{TR} / \mathrm{TE}=22 / 4 \mathrm{~ms}$, matrix $=256 \times 256$, flip angle $=30^{\circ}$, section thickness $=1.5 \mathrm{~mm}, \mathrm{FOV}=240 \mathrm{~mm}$ ), T2weighted images $(\mathrm{TR} / \mathrm{TE}=4000 \mathrm{~ms} / \mathrm{minimum}$ full $)$, and fluidattenuated inversion recovery images $(\mathrm{TR} / \mathrm{TE}=6000 / 140 \mathrm{~ms}$, matrix $=256 \times 256$, section thickness $=1 \mathrm{~mm}, \mathrm{FOV}=240 \mathrm{~mm}$ ) were obtained.

\section{ROls for CBT}

The ROIs (mean number of voxels in the ROI $=28 \pm 9$ ) were manually drawn under the direct supervision of a board-certified radiologist holding a Certificate of Added Qualification in Neuroradiology on the $\mathrm{B} 0$ images after inspection of the anatomic images, FA maps, and color FA maps. First, the "hand knob" of the primary motor cortex was identified. ${ }^{19} \mathrm{Next}$, an ROI for the face was placed in the primary motor cortex lateral to the hand knob in the axial and sagittal planes. ${ }^{16,19,20}$ The ROI for the tongue was then placed at the lowermost portion of the primary motor cortex just above the Sylvian fissure in the sagittal plane. ${ }^{16,18}$ The ROI for the pons was placed at the ventral part of the midpons through which the CBT travels, as manifested by 2 symmetric blue circles on the color FA maps to represent descending tracts. ${ }^{19}$ Anatomic images in the sagittal, coronal, and axial planes were used to confirm the final ROIs. The same seed ROIs were used for both deterministic and probabilistic tractography (Fig 1).

\section{Deterministic Tractography}

Deterministic tractography was performed by using the FACT algorithm implemented in DTI\&FiberTools. Whole-brain white matter masks were estimated and reconstructed by applying 2 thresholds to control the tracking area and minimize unwanted noise: a start and a stop mask. The start mask was set to FA $>0.25$, and mean diffusivity $<1.6 \times 10^{-3} \mathrm{~mm}^{2} / \mathrm{s}$, and the stop mask was set to FA $>0.1$ and mean diffusivity $<2 \times 10^{-3} \mathrm{~mm}^{2} / \mathrm{s}$. The maximum curvature of the tract was set to $60^{\circ}$. The whole-brain mask was then used to extract specific tracts between individual motor ROIs and the pons for each subject.

\section{Probabilistic Tractography}

Probabilistic tractography was performed by using DTI\&FiberTools in an extended Monte Carlo simulation method similar to the probabilistic index of connectivity method. ${ }^{6,7}$ Tracking areas were defined as regions with mean diffusivity $<2 \times 10^{-3} \mathrm{~mm}^{2}$ and FA $>0.1$. The number of random walks was set to 100,000 , and the maximum fiber length, to 150 voxels with no revisiting. ${ }^{7,11}$ The tracts were reconstructed by extraction of the most probable connected voxels between 2 ROIs. First, the visiting connectivity maps were generated for each ROI. ${ }^{7}$ For each visiting map, the frequency of a voxel reflects the degree of connectivity to the ROI. Second, the visiting maps from the 2 ROIs were combined to create a probability connectivity map that contains all voxels connecting to both ROIs. The probabilistic connectivity map represents the voxels with the highest likelihood of connecting any 2 selected ROIs. Visiting maps gener- 


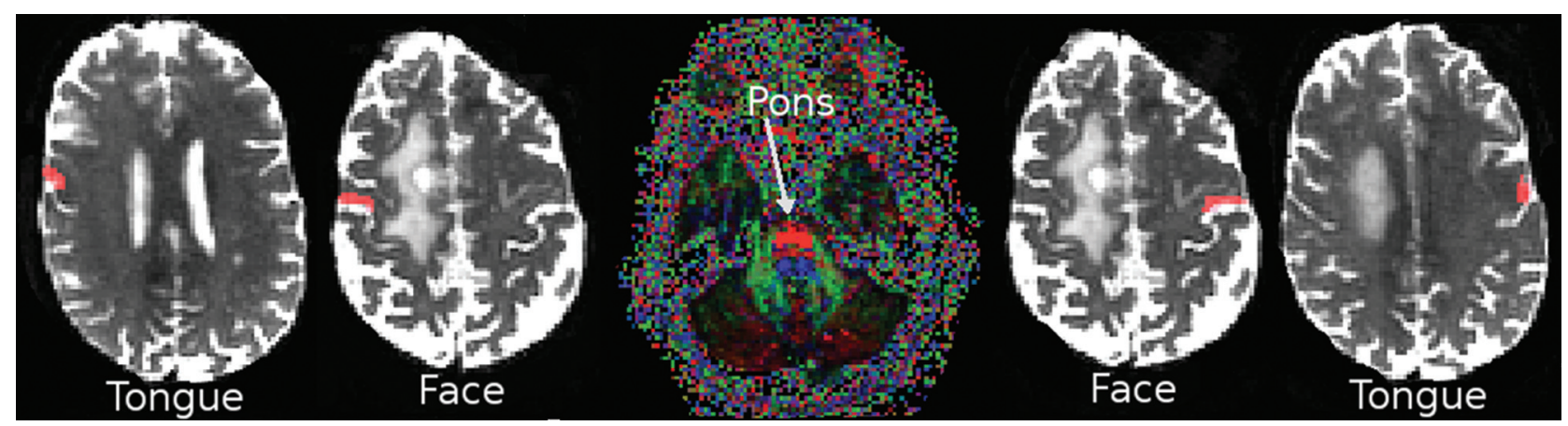

FIG 1. The location of ROIs for 1 patient with a right-handed tumor: Four seed ROIs in the precentral gyrus (primary motor cortex) of both hemispheres at the level of tongue and face homunculus and another in the pons were selected. Face and tongue ROls are located in the ipsilateral and contralateral primary motor cortex associated with the face and tongue, respectively. The ipsilateral or contralateral sides are in reference to the location of the tumor.

ated from the motor ROIs were separately combined with visiting maps from the pons ROI to produce 4 "probabilistic connectivity maps" for each subject, named as the following probabilistic connectivity maps: face ipsilateral to tumor, face contralateral to tumor, tongue ipsilateral to tumor, and tongue contralateral to tumor.

\section{Quantification of Probabilistic Connectivity}

Within the connectivity probabilistic maps, the voxels with the most connectivity were extracted by generating histograms of the connectivity between seed regions and applying a threshold $(0.001-0.5)$ to define the areas of highest connectivity. ${ }^{11}$ The number of voxels was then determined by counting voxels within each probabilistic connectivity map and normalizing them to the sum of their corresponding ROIs as shown below:

Number of Voxels $=$ Number of all Counted Voxels

in each Connectivity Map/(Motor ROI + Pons ROI).

The degree of connectivity of the probabilistic map or PIBI (probability index of forming part of the bundle of interest $)^{7}$ provides a measure of the strength of tract connectivity. It represents the probability that a voxel belongs to the white matter pathway connecting both ROIs. ${ }^{7}$ For each map, the mean value of the PIBI has been used to determine the degree of connectivity.

\section{Volumetric Measurements}

An averaged FA value of each probabilistic connectivity map was measured on the tumor and contralateral sides of each patient. The enhancing tumor was manually segmented on the contrast T1-weighted images by a neuroradiologist (with $>10$ years of experience) and transferred to the FA and B0 maps. The volume of the enhancing tumor and volumes of the connectivity maps were used to generate 3D volume-rendering images. Determinations of possible displacement (deviation of the tract route) and disruption $(P<.05 ;>95 \%$ decrease in the tract volume or interruption of tract route) were made by comparing the connectivity map on the tumor side with that of the normal side.

\section{Statistical Analysis}

Patients were stratified into 2 groups according to tumor distance from the primary motor cortex and/or CBT based on anatomic images. The margin of the enhancing tumor and/or peritumoral FLAIR/T2 abnormality $(n=23, \leq 1 \mathrm{~cm}$ and $n=27,>1 \mathrm{~cm}$ and $\leq 2 \mathrm{~cm} ; \leq 1 \mathrm{~cm}$ versus $>1 \mathrm{~cm}$ and $\leq 2 \mathrm{~cm}$ ) comparisons were made for the number of voxels, degree of connectivity, and the FA value of each connectivity map for both sides within each patient and across patients by using groups consisting of tumor and contralateral sides. Comparisons were also made between primary and secondary tumor types, to examine the differences from which possible infiltrative growth patterns are known to occur in primary tumors. The data were analyzed by using independent paired $t$ tests with statistical significance set at $P<.05$.

\section{RESULTS}

\section{Deterministic Tractography}

The entire course of the CBT between the face motor area and the pons or between the tongue motor area and the pons was not identified by using FACT tractography in any case on either the tumor or ipsilateral side. Instead, FACT tractography consistently terminated in regions adjacent to the tumor and/or areas with peritumoral abnormality with low FA $(<0.25)$ as denoted by orange arrows (Fig 2A). Tracts from seeds placed in the primary motor cortex (terminated before the internal capsule) and tracts from seeds placed in the pons traveled through the internal capsules and terminated at the level of the corona radiata before the expected lateral curves toward the face and tongue seeds (Fig 2A).

\section{Probabilistic Tractography}

On the other hand, probabilistic tractography successfully identified the CBT pathways from the face and tongue motor to the pons in all patients on both tumor and normal sides. The results of probabilistic tractography for 2 patients with tumor on different sides are shown in Fig $2 B,-C$.

\section{Patients with Tumors Located Less Than 1 Centimeter from the Anatomic Location of the Corticobulbar Tract}

Within patients, although the tumor side trended toward an increased mean number of voxels (face, $+16 \%$ and tongue, $+2 \%$ ) and mean connectivity values (face, $+15 \%$ and tongue, $+15 \%$ ), as summarized in Table 1, no significant differences were observed between the tumor and normal sides in the number of 


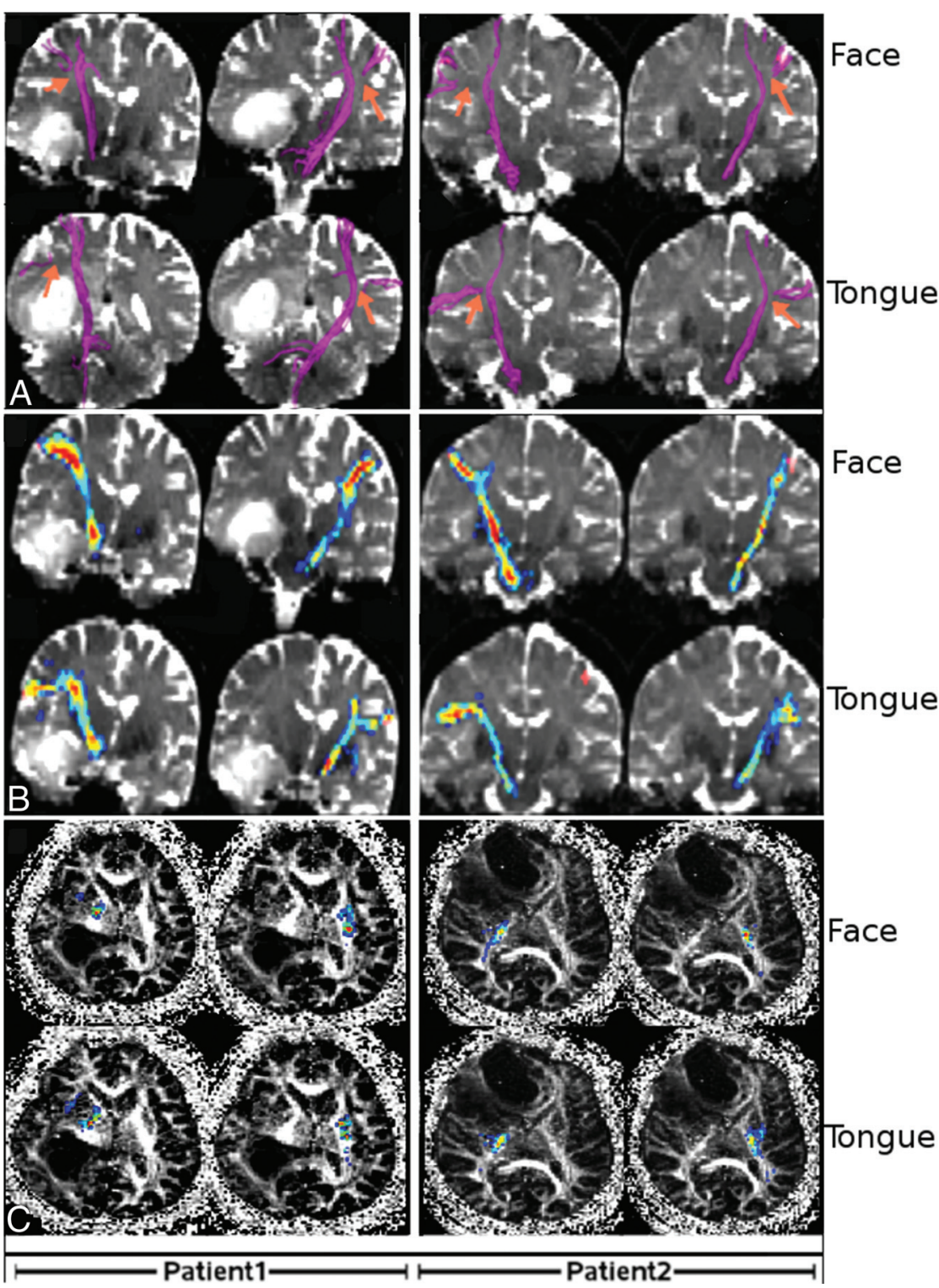

FIG 2. FACT and probabilistic fiber tractography results obtained from 2 patients: 1) a 56-year-old man with glioblastoma, and 2) a 63-year-old woman with brain metastasis (adenoid cystic carcinoma), A, The FACT algorithm was not capable of extracting fibers between the pons and face motor or pons and tongue motor. The FACT tracts were not continuous in areas where the CBT crosses the much more prominent superior longitudinal fasciculus (orange arrow). $B$, The probabilistic method was successful in identifying the CBT pathways from the face and tongue motor areas to the pons. $C$, The probabilistic connectivity of the face and tongue at the level of internal capsule. Colors represent the degree of connectivity: from 0.001 in blue to 0.5 in red. voxels or degree of connectivity along each connectivity map $(P<.13)$. For each patient, the tumor side showed significantly lower FA values of the probability maps of both face and tongue compared with the ipsilateral side $(P<$ .001 and $P<.003$, respectively). The mean FA value across patients was also lower on the tumor side (face, $-11 \%$ and tongue, $-11 \%)$, as summarized in Table 1.

\section{Patients with Tumors Located More Than 1 Centimeter and Equal to or Less Than 2 Centimeters from the Anatomic Location of the Corticobulbar Tract}

Across patients, the tumor side showed a trend toward increased mean voxel number (face, $+26 \%$ and tongue, $+7 \%$ ) (Table 2) and face connectivity values $(+23 \%)$, but decreased tongue connectivity values $(-6 \%)$ (Table 2$)$; however, these differences were not significant $(P<.08)$. In addition, the probabilistic connectivity map of the face and tongue on the tumor side had lower mean FA values of the connectivity maps (face, $P<.03$, and tongue, $P<.016)$ (Table 2 ).

\section{Types of Tumors}

When tumor type was stratified as primary tumors $(n=31)$ versus secondary tumors $(n=19)$, no significant differences were observed among tracts in terms of the mean FA $(P<.6)$, number of voxels $(P<.2)$, or connectivity values $(P>.1)$ (On-line Table).

\section{Clinical Visualization}

Visual inspection showed continuous connectivity maps for the face and tongue motor tracts from the primary

Table 1: Data for patients with tumor $<1 \mathrm{~cm}$ from the corticobulbar tract

\begin{tabular}{|c|c|c|c|c|c|c|c|c|}
\hline \multirow{2}{*}{$\begin{array}{l}\text { Averaged over } \\
\text { Subjects } \pm \text { SD }\end{array}$} & \multicolumn{4}{|c|}{ Connectivity Map of Face Motor } & \multicolumn{4}{|c|}{ Connectivity Map of Tongue Motor } \\
\hline & Tumor & Nontumor & $P$ Value & $\%$ Change & Tumor & Nontumor & $P$ Value & \% Change \\
\hline No. of voxels & $10.8555 \pm 4.3837$ & $9.3350 \pm 2.9498$ & .1300 & $+16 \%$ & $12.0866 \pm 5.8446$ & $11.8514 \pm 4.3694$ & .8500 & $+2 \%$ \\
\hline Degree of connectivity & $0.6768 \pm 0.3906$ & $0.5884 \pm 0.3662$ & .3500 & $+15 \%$ & $0.6367 \pm 0.347$ & $0.6357 \pm 0.349$ & .9900 & $+0.15 \%$ \\
\hline FA value & $0.4615 \pm 0.0664$ & $0.5211 \pm 0.0352$ & .0000 & $-11 \%$ & $0.4557 \pm 0.0701$ & $0.5104 \pm 0.045$ & .0003 & $-11 \%$ \\
\hline
\end{tabular}

Table 2: Data for patients with tumor $>1 \mathrm{~cm}$ from the corticobulbar tract

\begin{tabular}{|c|c|c|c|c|c|c|c|c|}
\hline \multirow{2}{*}{$\begin{array}{l}\text { Averaged over } \\
\text { Subjects } \pm \text { SD }\end{array}$} & \multicolumn{4}{|c|}{ Connectivity Map of Face Motor } & \multicolumn{4}{|c|}{ Connectivity Map of Tongue Motor } \\
\hline & Tumor & Nontumor & $P$ Value & $\%$ Change & Tumor & Nontumor & $P$ Value & \% Change \\
\hline No. of voxels & $10.9157 \pm 4.7361$ & $8.6965 \pm 3.3798$ & .0800 & $+26 \%$ & $13.6748 \pm 7.1344$ & $12.7478 \pm 6.8817$ & .3900 & $+7 \%$ \\
\hline Degree of connectivity & $0.59 \pm 0.4437$ & $0.4799 \pm 0.2992$ & .3200 & $+23 \%$ & $0.6267 \pm 0.3798$ & $0.6645 \pm 0.3687$ & .6400 & $-6 \%$ \\
\hline FA value & $0.4924 \pm 0.0567$ & $0.5191 \pm 0.0338$ & .0296 & $-5 \%$ & $0.4765 \pm 0.0650$ & $0.5118 \pm 0.0537$ & .0158 & $-7 \%$ \\
\hline
\end{tabular}




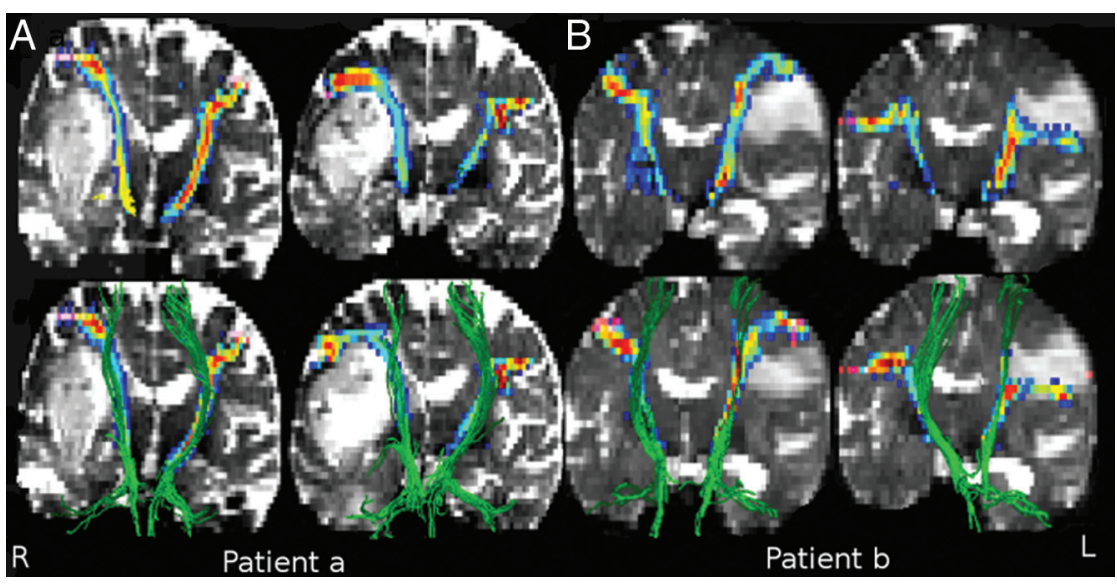

FIG 3. Probabilistic-versus-FACT tractography of 2 patients. $A, \mathrm{~A} 69$-year-old man with glioblastoma located $<1 \mathrm{~cm}$ from the CBT. B, A 67-year-old woman with brain metastasis from lung carcinoma located $>1$ and $\leq 2 \mathrm{~cm}$ from the CBT. First row: probabilistic tractography results of the connectivity maps of the face and tongue motor areas. respectively. Second row: probabilistic-versus-FACT tractography. Probabilistic connectivity is shown as multicolored maps, and FACT is shown as green fiber projections. The FACT tract discontinuation is visible. Colors represent the degree of connectivity, from 0.001 in blue to 0.5 in red.

motor cortex to the pons. Separate pathways originating from face and tongue seeds consistently converged at the level of the internal capsule with decreasing connectivity values. Tumors along the path of the probabilistic maps resulted in mass effect and deflection of the maps, which appeared to wrap around the tumor, but remained continuous (Fig 3).

\section{DISCUSSION}

We demonstrate that the probabilistic tractography method of analysis of DTI data can successfully identify the CBT pathways from the face and tongue regions of the primary motor cortex to the pons in patients with brain tumors. This method is in contrast to deterministic tractography, in which the CBT pathways could not be identified, even on the side contralateral to the tumor. Our results suggest that probabilistic tractography can be used to visualize the CBT pathways, to guide neurosurgical planning, and to minimize the risk of white matter damage during the operation.

Preliminary effort to visualize the CBT motor pathways of the face and tongue has been described by a few groups, but their results were limited by the number of subjects studied. Akter et $\mathrm{al}^{17}$ used multitensor tractography to detect face and tongue motor pathways in 5 healthy adults, but their techniques required complicated data processing and a longer postprocessing time compared with the usual single tensor tractography. A multitensor tractography study with high-angular-resolution diffusion imaging by Yamada et $\mathrm{al}^{18}$ depicted face and tongue motor pathways; however, the CBT tracts of the face were identified in only $70 \%$ of their patients. Another study ${ }^{15}$ described 2-tensor tractography as the preferred method for detecting hand and face fibers compared with single-tensor and probabilistic tractography in 2 controls and 1 patient. Kreher et $\mathrm{al}^{7}$ showed that probabilistic fiber tracking could extract fibers of the entire motor cortex; however, they only demonstrated this in control adults and not in patients with tumors. In this study, we compared the ability of both the deterministic FACT and the probabilistic fiber tracking methods to extract CBT pathways associated with face and tongue motor in 50 patients with brain tumor. In addition, we also compared the results on the basis of the distance between the tumor margin and motor cortex.

We found that the number of voxels, a measure of the volume of the probabilistic map of connectivity, was increased on the side of the tumor ( $\mathrm{Ta}$ bles 1 and 2). We postulate that this increase in volume was due to deflection and stretching of the CBTs by mass effect from the tumors and peritumoral abnormalities. Similar results have been described by Yeh et al, ${ }^{1}$ who described altered morphology of the corticospinal tract with stretching of the tracts at the internal capsule. This deflection of the CBT was seen near the tumor in all cases.

The mean degree of connectivity of probabilistic maps was also slightly (15\%) increased on the tumor side (Tables 1 and 2). Although this increase was not significant, it may be because of compression of the CBT due to mass effect, with subsequent increased fiber attenuation at tractography as discussed by Young et al. ${ }^{21}$ Similar to that in other groups, ${ }^{1-4} \mathrm{FA}$ was decreased in tracts associated with brain tumors. This decrease in FA or increase in the degree of connectivity is a primary limitation of tractography techniques, which become less accurate due to uncertainties in determining the principal directionality of each voxel. The increases in the number of voxels occurred despite the decreased FA values; this finding suggests that probabilistic tractography may be reliable in visualizing important white matter tracts affected by brain tumors.

Visualization of the normal CBT pathways for the face and tongue shows a descending course from the lateral portions of the primary motor cortex into the medial portions of the pons. This is consistent with previous anatomic probabilistic tractography studies that demonstrated alterations in the orientation of the descending motor fibers. ${ }^{15,16}$ In particular, a study by Pan et al ${ }^{16}$ showed that the CBT fibers either cross or have crossed medial to the CST fibers at the level of the posterior limb of the internal capsule. These descending medial courses were also visible on the tumor sides, though there were additional deflections caused by tumor and peritumoral abnormalities.

This study may have the following limitations. First, we relied on anatomic markers for placing the seed ROIs for the face and tongue in the primary motor cortex. Although seed ROIs drawn from voxels activated by functional MR imaging can provide optimal localization of the correct locations, these data were not available in our retrospective cohort. Second, the probabilistic approach can sometimes introduce uncertainties, especially when measuring characteristics of the fiber bundle such as the length and degree of deflection by using the probabilistic map of connectivity of the face and tongue on the tumor side. ${ }^{7}$ 


\section{CONCLUSIONS}

Probabilistic tractography can successfully visualize the face- and tongue-associated CBT pathways in patients with brain tumors. By contrast, deterministic tractography was not successful in reconstructing the CBT on the tumor or contralateral sides. Probabilistic tractography may be useful to guide preoperative planning for patients with brain tumors affecting or adjacent to the CBT.

Disclosures: Robert J. Young—RELATED: Grant: Radiological Society of North America, ${ }^{*}$ Comments: 2011 Fujifilm Medical Systems/Radiological Society of North America. Research Seed Grant. *Money paid to the institution.

\section{REFERENCES}

1. Yeh YY, Huang SC, Chiang WY, et al. Effects of brain tumor on corticospinal tract and motor function: analysis of tract stretch using diffusion spectrum imaging tractography. In: Proceedings of the Annual Meeting of the International Society for Magnetic Resonance in Medicine, Honolulu, Hawaii. April 18-24, 2009:17

2. Jellison BJ, Field AS, Medow J, et al. Diffusion tensor imaging of cerebral white matter: a pictorial review of physics, fiber tract anatomy, and tumor imaging patterns. AJNR Am J Neuroradiol 2004; 25:356-69

3. Parmar H, Sitoh YY, Yeo TT. Combined magnetic resonance tractography and functional magnetic resonance imaging in evaluation of brain tumors involving the motor system. J Comput Assist Tomogr 2004;28:551-56

4. Mori S, Crain BJ, Chacko VP, et al. Three-dimensional tracking of axonal projections in the brain by magnetic resonance imaging. Ann Neurol 1999;45:265-69

5. Basser PJ, Pajevic S, Pierpaoli C, et al. In vivo fiber tractography using DT-MRI data. Magn Reson Med 2000;44:625-32

6. Parker JG, Haroon HA, Wheeler-Kingshott CA. A framework for a streamline-based probabilistic index of connectivity (PICo) using a structural interpretation of MRI diffusion measurements. J Magn Reson Imaging 2003;18:242-54

7. Kreher BW, Schnell S, Mader I, et al. Connecting and merging fibres: pathway extraction by combining probability maps. Neuroimage 2008;43:81-89

8. Lazar M, Weinstein JS, Tsuruda KM, et al. White matter tractography using diffusion tensor deflection. Hum Brain Mapp 2003;18: 306-21

9. Parker GJ, Alexander DC. Probabilistic Monte Carlo based mapping of cerebral connections utilising whole-brain crossing fibre information. Inf Process Med Imaging 2003;18:684-95

10. Li Z, Peck KK, Brennan N, et al. Diffusion tensor tractography of the arcuate fasciculus in patients with brain tumors: comparison between detrministic and probabilistic models. J Biomed Sci Eng 2013;6:192-200q

11. Jenabi M, Peck KK, Young RJ, et al. Probabilistic fiber tracking of the language and motor white matter pathways of the supplementary motor area (SMA) in patients with brain tumors. J Neuroradiol 2014;41:342-49

12. Nguyen-Thanh T, Reisert M, Anastasopoulos C, et al. Global tracking in human gliomas: a comparison with established tracking methods. Clin Neuroradiol 2013;23:263-75

13. Behrens TE, Berg HJ, Jbabdi S, et al. Probabilistic diffusion tractography with multiple fibre orientations: what can we gain? Neuroimage 2007;34:144-55

14. Mader I, Nguyen Thanh T, Schnell S, et al. Probability maps compared to face algorithm in human gliomas. In: Proceedings of the Annual Meeting of the International Society for Magnetic Resonance in Medicine, Stockholm, Sweden. May 1-7, 2010:18

15. Qazi AA, Radmanesh A, O'Donnell L, et al. Resolving crossings in the corticospinal tract by two-tensor streamline tractography: method and clinical assessment using fMRI. NeuroImage 2009;47: T98-106

16. Pan C, Peck KK, Young RJ, et al. Somatotopic organization of motor pathways in the internal capsule: a probabilistic diffusion tractography study. AJNR Am J Neuroradiol 2012;33:1274-80

17. Akter M, Hirai T, Sasao A, et al. Multi-tensor tractography of the motor pathway at 3T: a volunteer study. Magn Reson Med Sci 2011;101:59-63

18. Yamada K, Sakai K, Hoogenraad FGC, et al. Multitensor tractography enables better depiction of motor pathways: initial clinical experience using diffusion-weighted MR imaging with standard $\mathbf{b}$ value. AJNR Am J Neuroradiol 2007;28:1668-73

19. Yousry TA, Schmid UD, Alkadhi H, et al. Localization of the motor hand area to a knob on the precentral gyrus: a new landmark. Brain 1997;120:141-57

20. McCarthy G, Allison T, Spencer DD. Localization of the face area of human sensorimotor cortex by intracranial recording of somatosensory evoked potentials. J Neurosurg 1993;79:874-84

21. Young RJ, Lee V, Peck KK, et al. Diffusion tensor imaging and tractography of the corticospinal tract in the presence of enlarged Virchow-Robin spaces. J Neuroimaging 2014;24:79-82 